\title{
A $k-\epsilon$ MODEL OF THE LOWER OVERSHOOT LAYER
}

\author{
K. PETROVAY \\ Instituto de Astrofísica de Canarias \\ E-38200 La Laguna, Tenerife, Spain
}

One region where discrepancies between the seismic and standard solar models continue to exist is the overshoot layer below the convective zone (Gough et al. 1996). A main reason for this discrepancy is clearly the lack of a realistic physical model for astrophysical overshoot. Beside numerical experiments, the most promising approach to the construction of such a model is based on the Reynolds-stress formalism of turbulence theory (Speziale 1991). As a first step toward the development of a more complete Reynolds stress model of the overshoot, here we present a simple $k-\epsilon$ model, valid under the following conditions:

(a). Incompressibility: $\partial_{i} v_{i}=0$. Owing to the short length scale (cf. Fig. 1), this assumption is somewhat better founded here than in other models.

(b). Closure: Let $\overline{w v_{i}^{2}} / 2=C_{w k} k^{3 / 2}$ and $\overline{w \epsilon}=C_{w \epsilon} k^{1 / 2} \bar{\epsilon} .\left(w=v_{z}\right.$ vertical velocity; $k \equiv V^{2} / 2$ : kinetic energy; $\epsilon$ : dissipation rate). Now assume $C_{w k}=$ const., $C_{w \epsilon}=$ const. This is warranted if $l / H_{V} \simeq$ constant $\left(H_{V}\right.$ : scale height of $\left.V^{2}\right)$, which will be seen indeed to be the case in our model, cf. eqs. (3)-(4).

(c). No correlation with thermodynamic fluctuations: $\overline{v_{i} \rho}=\overline{v_{i} P}=\overline{v_{i} T}=0$. The assumption implies that these correlations vanish in a very thin $\left(\ll H_{P}\right)$ transition layer below $\Delta \nabla=0$. No change in the geometrical flow properties is expected in such a thin layer, so $l_{0}, C_{w k}$, and $C_{w \epsilon}$ should be the same as in the lower part of the unstable layer. On the basis of numerical experiments here we use $l_{0}=H_{P}$, $C_{w^{\prime} k}=0.6, C_{w^{\prime} \epsilon}=3, q=2.4$. (The index ' 0 ' refers to values at $z=0$ i.e. at $\Delta \nabla=0$.) Note however that $V$ may be significantly reduced in the transition layer, so $V_{0}$ (and thus $\left.\bar{\epsilon}_{0}\right)$ is undetermined in the present model.

The fluctuating part of the equation of motion now reads

$$
\partial_{t} v_{i}+v_{j} \partial_{j} v_{i}=-\partial_{i} P^{\prime} / \rho+\rho^{\prime} g_{i} / \rho+\nu \partial_{j}^{2} u_{i}
$$

With the above assumptions, the first moment of (1) with $v_{i}$ yields:

$$
\partial_{j}\left(k^{3 / 2}\right)=-\bar{\epsilon} / C_{w k} .
$$

In all previous models of the overshoot layer the length scale $l=V^{3} / \bar{\epsilon}$ was set arbitrarily. Here in contrast we perform the operation $2 \nu \overline{\left(\partial_{k} v_{i}\right) \partial_{k}}$ on eq. (1):

$$
\partial_{j}\left(k^{1 / 2} \bar{\epsilon}\right)=2 \nu^{2} \overline{\left(\partial_{j} \partial_{k} v_{i}\right)^{2}} / C_{w \epsilon}=-\left(C_{\epsilon} / C_{w \epsilon}\right) \bar{\epsilon}^{2} / k
$$




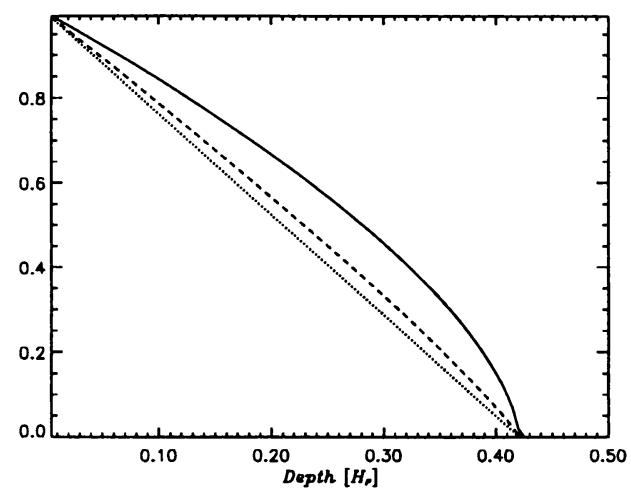

Figure 1. Normalized values of the turbulent velocity $V$ (solid), dissipation rate $\bar{\epsilon}$ (dashed) and length scale $l$ (dotted) as functions of $z$ in a plane parallel model

where $C_{t} \simeq 1.8$ from experiments and RNG theory. In the plane-parallel limit then

$$
d_{z}\left(V^{3}\right)=-2 \sqrt{2} \bar{\epsilon} / C_{w k} \quad d_{z}(V \bar{\epsilon})=-2 \sqrt{2}\left(C_{\epsilon} / C_{w \epsilon}\right) \bar{\epsilon}^{2} / V^{2}
$$

and this system admits the analytic solution (Fig. 1)

$$
\begin{gathered}
\bar{\epsilon} / \bar{\epsilon}_{0}=\left(V / V_{0}\right)^{q-1} \\
V^{4-q}=V_{0}^{1-q}\left(V_{0}^{3}-\frac{2^{3 / 2}}{3} \frac{4-q}{C_{w^{\prime} k}} \epsilon_{0} z\right) \quad q=3 C_{\epsilon} \frac{C_{w^{\prime} k}}{C_{w \epsilon}} .
\end{gathered}
$$

It is apparent that $l=V^{3} / \bar{\epsilon}$ decreases linearly with depth $z$, i.e. the widely used assumption $l \sim H_{P}$ is indeed very unrealistic. The overshoot distance in the kinematic (i.e. $V \neq 0$ ) sense is of order $H_{P}$, its exact value being rather sensitive to $C_{w^{\prime} k}$ and $C_{w \epsilon}: z_{\mathrm{ov}} / l_{0}=3 / 2^{3 / 2} C_{w k} /(4-q)$.

In conclusion, this illustrative model shows that the length parameter can be determined self-consistently. Fitting the model to the results of numerical experiments on deep turbulent convection yields $z_{\mathrm{ov}} \sim H_{P}$ for the kinematic extent of overshoot. On the other hand, the overshoot in thermal sense (i.e. the quasiadiabatic part of the subadiabatic layer) is negligible in this model (as a direct consequence of assumption (c) above). This could help in explaining the remaining discrepancies between observed and calculated oscillation frequencies, and it would also be beneficial for the dynamo.

The practical value of the model hinges upon assumption (c), which should be tested in a more complete model including thermodynamic correlations.

This work was funded by the DGES project no. 95-0028, by the OTKA grant no. 016196, and by the FKFP grant no. 0201/97.

\section{References}

Gough D. O. et al. 1996, Science, 272, 1296

Speziale, C. G. 1991, Ann. Rev. Fluid Mech., 23, 107 